\title{
ON THE IMAGE OF MRC FIBRATIONS OF PROJECTIVE MANIFOLDS WITH SEMI-POSITIVE HOLOMORPHIC SECTIONAL CURVATURE
}

\author{
SHIN-ICHI MATSUMURA
}

\begin{abstract}
In this paper, we pose several conjectures on structures and images of maximal rationally connected fibrations of smooth projective varieties admitting semi-positive holomorphic sectional curvature. Toward these conjectures, we prove that the canonical bundle of images of such fibrations is not big. Our proof gives a generalization of Yang's solution using RC positivity for Yau's conjecture. As an application, we show that any compact Kähler surface with semi-positive holomorphic sectional curvature is rationally connected, or a complex torus, or a ruled surface over an elliptic curve.
\end{abstract}

\section{INTRODUCTION}

One of the famous conjectures, which were posed by S.-T. Yau in [Yau82], states that any compact Kähler manifold with negative (resp. positive) holomorphic sectional curvature has an ample canonical bundle (resp. is rationally connected).

The former conjecture was affirmatively solved for projective varieties of dimension $\leq 3$ in [HLW10], solved for projective varieties of arbitrary dimension in [WY16], and solved for compact Kähler manifolds in [TY15]. On the other hand, it is known that a smooth projective variety whose holomorphic sectional curvature is identically zero admits a finite étale cover by an abelian variety (see [HLW16, Proposition 2.2], [Ber66], [Igu54]). In their paper [HLWZ17], Heier-Lu-Wong-Zheng showed that any smooth projective variety with semi-negative holomorphic sectional curvature admits a finite étale cover by the product of an abelian variety and a projective variety with ample canonical bundle, under the assumption of the abundance conjecture (see also [HLW16]).

The latter conjecture on positive holomorphic sectional curvature was affirmatively solved for projective varieties in [HW15] and solved for compact Kähler manifolds in [Yan18a]. Therefore one of the remaining most interesting problems in this field is to determine a structure of smooth projective varieties with "semi-positive" holomorphic sectional curvature.

Date: May 24, 2022, version 0.01.

2010 Mathematics Subject Classification. Primary 32Q10, Secondary 53C25, 14M22.

Key words and phrases. Holomorphic sectional curvatures, Maximal rationally connected fibrations, Rationally connectedness, Abelian varieties, Ruled surfaces, Partially positive curvatures, RC positivity, Vanishing theorems, Minimal models. 
In this paper, we pose the following conjecture (Conjecture 1.1) by focusing on the similarity to semi-negative holomorphic sectional curvature. This conjecture also can be seen as a generalization of the structure theorem for (holomorphic) bisectional curvature proved in [HSW81] and [Mok88] to holomorphic sectional curvature (see also [CG71] and [CG72]). As a new approach to rational connectedness, Yang introduced the notation of RC positivity in the breakthrough paper [Yan18a]. Toward Conjecture 1.1, we study maximal rationally connected (MRC for short) fibrations of smooth projective varieties with semipositive holomorphic sectional curvature, by developing the theory of RC "semi"-positivity.

Conjecture 1.1. Let $X$ be a compact Kähler manifold with semi-positive holomorphic sectional curvature. Then there exists a smooth morphism $X \rightarrow Y$ such that a fiber is rationally connected and $Y$ admits a finite étale cover $A \rightarrow Y$ by an abelian variety $A$.

For a smooth projective variety $X$ with semi-positive holomorphic sectional curvature, it seems to be quite difficult to directly confirm that $X$ has positive irregularity (in other words, its Albanese map is non-trivial). On the other hand, it can be shown that a MRC fibration $X \rightarrow Y$ of $X$ is non-trivial (that is, $0<\operatorname{dim} Y<\operatorname{dim} X$ ) when $X$ is neither rationally connected nor an abelian variety up to finite étale covers.

In this paper, we attempt to approach Conjecture 1.1 by investigating a MRC fibration $X \rightarrow Y$ of $X$ instead of the Albanese map (see [Cam92], [KoMM92] for MRC fibrations and rationally connectedness). Note that the image $Y$ of $\mathrm{MRC}$ fibrations is determined up to birational equivalence (in particular, we may assume that $Y$ is smooth by taking a resolution of singularities), and also that the image $Y$ is not uniruled by [GHS03, Theorem 1.1] (equivalently, the canonical bundle $K_{Y}$ of $Y$ is pseudo-effective by [BDPP13]).

From the viewpoint of Conjecture 1.1, it is natural to expect that a minimal model of the image $Y$ (if exists) admits a finite étale cover by an abelian variety. Further it can also be expected that a MRC fibration of $X$ to a minimal model of $Y$ is actually a smooth morphism. For this purpose, it seems to be the first step to show that the numerical dimension of the image $Y$ is zero. Based on the above observations, we pose the following two conjectures :

Conjecture 1.2. Let $X$ be a smooth projective variety with semi-positive holomorphic sectional curvature, and let $X \rightarrow Y$ be a dominant rational map from $X$ to a smooth projective variety $Y$ with the pseudo-effective canonical bundle $K_{Y}$. Then the numerical dimension $\nu(Y)=\nu\left(K_{Y}\right)$ is equal to zero. In particular, the numerical dimension of the image of non-trivial MRC fibrations of $X$ is zero. (See [Nak] for the definition of the numerical dimension $\nu(\cdot)$.)

Conjecture 1.3. Let $X$ be a smooth projective variety with semi-positive holomorphic sectional curvature, and let $X \rightarrow Y$ be a MRC fibration of $X$ to a projective variety $Y$. If $Y$ has (at most) terminal singularities and the canonical divisor $K_{Y}$ is a nef $\mathbb{Q}$-Cartier divisor (that is, $Y$ is a minimal model), then $Y$ is smooth and $f$ is a morphism. Moreover $Y$ admits a finite étale cover by an abelian variety. 
In this paper, we prove that the canonical bundle $K_{Y}$ in Conjecture 1.2 is not a big line bundle (that is, $\nu(Y)<\operatorname{dim} Y$ ). For the proof, we develop the theory of RC positivity introduced in [Yan18a] (in particular RC semi-positivity). (See [Yan18b] and [Yan18c] for the recent development of RC-positivity.) Our argument in the proof can be seen as a generalization of the solution for Yau's conjecture proved in [Yan18a].

Theorem 1.4. Let $X$ be a compact Kähler manifold with semi-positive holomorphic sectional curvature, and let $\phi: X \rightarrow Y$ be a dominant meromorphic map from $X$ to a smooth projective variety $Y$. Then $K_{Y}$ is not a big line bundle (that is, $\left.\nu(Y)<\operatorname{dim} Y\right)$.

As a corollary of Theorem 1.4, we obtain the following result, which affirmatively solves Conjecture 1.3 for smooth projective surfaces (even for compact Kähler surfaces).

Corollary 1.5. Let $X$ be a compact Kähler surface with semi-positive holomorphic sectional curvature. Then one of the followings holds:

- $X$ is rationally connected.

- $X$ is a complex torus.

- $X$ is a ruled surface over an elliptic curve.

In particular, Conjecture 1.3 is true for compact Kähler surfaces with semi-positive holomorphic sectional curvature.

In Section 2, we will recall some results on curvatures of vector bundles and the notion of RC positivity. In Section 3, we will prove Theorem 1.4 and its corollary.

In this paper, we interchangeably use the words "line bundles", "invertible sheaves", and "Cartier divisors" (also "vector bundles" and "locally free sheaves"). Further we denote by the notation $D^{\otimes m}$ the $m$-th multiple $m D$ of a divisor $D$. Note that we treat only the holomorphic sectional curvature obtained from Kähler metrics throughout this paper.

One year after the previous version of this paper had been put in arXiv, Conjecture 1.1 was affirmatively solved in a "strong" form for smooth projective varieties in [Mat18a] and [Mat18b]. This paper is a modified and shorten manuscript. In the previous version, it is shown that the numerical dimension of $K_{Y}$ is equal to zero in Theorem 1.4 under the assumption that $Y$ admits a good minimal model (which is true when $\operatorname{dim} Y \leq 3$ ) by developing the techniques in this paper. In [Mat18a] and [Mat18b], the strategy explained in this paper turns out well.

\section{Preliminaries}

2.1. Curvature of vector bundles. In this subsection, we fix the notation for various curvatures and recall some curvature formulas for induced metrics.

Let $E$ be a (holomorphic) vector bundle of rank $r$ on a complex manifold $X$ of dimension $n$ and let $g=\sum_{k, \ell} g_{k \bar{l}} e_{k}^{\vee} \otimes \bar{e}_{\ell}^{\vee}$ be a (smooth) hermitian metric on $E$. For the hermitian vector bundle $(E, g)$, the Chern curvature

$$
\sqrt{-1} \Theta_{g}:=\sqrt{-1} \Theta_{(E, g)} \in C^{\infty}\left(X, \Lambda^{1,1} \otimes \operatorname{End}(E)\right)
$$


is defined by

$$
\sqrt{-1} \Theta_{g}\left(\partial / \partial z_{i}, \partial / \partial \bar{z}_{j}\right)\left(e_{k}\right):=\sqrt{-1}\left(-\frac{\partial^{2} g_{k \bar{\alpha}}}{\partial z_{i} \partial \bar{z}_{j}} g^{\alpha \bar{\ell}}+\frac{\partial g_{k \bar{\alpha}}}{\partial z_{i}} g^{\alpha \bar{\beta}} \frac{\partial g_{\beta \bar{\gamma}}}{\partial \bar{z}_{j}} g^{\gamma \bar{\ell}}\right) e_{\ell},
$$

where $\left(z_{1}, z_{2}, \ldots, z_{n}\right)$ is a local coordinate of $X$ and $\left\{e_{i}\right\}_{i=1}^{r}$ is a local frame of $E$. Here we used the Einstein convention for the summation.

Let $\Lambda^{m} E$ denote the vector bundle defined by the $m$-th exterior product of $E$. The hermitian metric $g$ on $E$ induces the hermitian metric $\Lambda^{m} g$ on $\Lambda^{m} E$. It is easy to see that the Chern curvature $\sqrt{-1} \Theta_{\Lambda^{m} g}=\sqrt{-1} \Theta_{\left(\Lambda^{m} E, \Lambda^{m} g\right)}$ of $\left(\Lambda^{m} E, \Lambda^{m} g\right)$ satisfies that

$$
\sqrt{-1} \Theta_{\Lambda^{m} g}(v, \bar{w})\left(a_{1} \wedge a_{2} \wedge \cdots \wedge a_{m}\right)=\sum_{j=1}^{m}\left(a_{1} \wedge \cdots \wedge \sqrt{-1} \Theta_{g}(v, \bar{w})\left(a_{j}\right) \wedge \cdots \wedge a_{m}\right)
$$

for any tangent vectors $v, w$ in the (holomorphic) tangent bundle $T_{X}$ and any vector $a_{i}$ in $E$. Similarly, it can be seen that the hermitian metric $S^{\ell} g$ on the $\ell$-th symmetric product $S^{\ell} E$ of $E$ induced by $g$ satisfies that

$$
\sqrt{-1} \Theta_{S^{\ell} g}(v, \bar{w})\left(a_{1} \odot a_{2} \odot \cdots \odot a_{\ell}\right)=\sum_{j=1}^{\ell}\left(a_{1} \odot \cdots \odot \sqrt{-1} \Theta_{g}(v, \bar{w})\left(a_{j}\right) \odot \cdots \odot a_{\ell}\right)
$$

for any tangent vectors $v, w \in T_{X}$ and any vectors $a_{i} \in E$. Further, for a hermitian vector bundle $(F, h)$, it can also be seen that the induced hermitian metric $g \otimes h$ on $E \otimes F$ satisfies that

$$
\sqrt{-1} \Theta_{g \otimes h}(v, \bar{w})(a \otimes b)=\sqrt{-1} \Theta_{g}(v, \bar{w})(a) \otimes b+a \otimes \sqrt{-1} \Theta_{h}(v, \bar{w})(b)
$$

for any tangent vectors $v, w \in T_{X}$ and any vectors $a \in E$ and $b \in F$.

The curvature tensor

$$
R_{g}=R_{(E, g)} \in C^{\infty}\left(X, \Lambda^{1,1} \otimes E^{\vee} \otimes \bar{E}^{\vee}\right)
$$

is defined to be

$$
R_{g}(v, \bar{w}, e, \bar{f}):=\left\langle\sqrt{-1} \Theta_{g}(v, \bar{w})(e), f\right\rangle_{g}
$$

for tangent vectors $v, w \in T_{X}$ and vectors $e, f \in E$. Throughout this paper, the notation $E^{\vee}$ denotes the dual vector bundle of $E$ and $\langle\bullet, \bullet\rangle_{g}$ denotes the inner product with respect to $g$. When $E$ is the tangent bundle $T_{X}$ and $g$ is a hermitian metric on $T_{X}$, the holomorphic sectional curvature $H_{g}$ is defined to be

$$
H_{g}([v]):=\frac{R_{g}(v, \bar{v}, v, \bar{v})}{|v|_{g}^{4}}
$$

for a non-zero tangent vector $v \in T_{X}$, which can be seen as a smooth function on the projective space bundle $\mathbb{P}\left(T_{X}^{\vee}\right)$ (that is, the set of all complex lines $[v]$ in $T_{X}$ ). The holomorphic section curvature is called positive (resp. semi-positive) if $H_{g}([v])>0$ (resp. $\left.H_{g}([v]) \geq 0\right)$ holds for any non-zero tangent vector $v \in T_{X}$. We remark that there exists the minimum value of $H_{g}$ on $\mathbb{P}\left(T_{X, p}^{\vee}\right)$ at every point $p \in X$ by compactness of $\mathbb{P}\left(T_{X, p}^{\vee}\right)$. 
PROJECTIVE MANIFOLDS WITH SEMI-POSITIVE HOLOMORPHIC SECTIONAL CURVATURE 5

If $g$ is a Kähler metric (that is, the associated $(1,1)$-form $\omega_{g}$ is $d$-closed), the following symmetry holds:

$$
R_{g}\left(e_{i}, \bar{e}_{j}, e_{k}, \bar{e}_{\ell}\right)=R_{g}\left(e_{k}, \bar{e}_{\ell}, e_{i}, \bar{e}_{j}\right)=R_{g}\left(e_{k}, \bar{e}_{j}, e_{i}, \bar{e}_{\ell}\right) .
$$

From the above symmetry, we can obtain Royden's lemma (see [Roy80]) and a refinement of [Yan17, Lemma 4.1], which play a crucial role in the proof of Theorem 1.4.

Lemma 2.1 ([Roy80]). Let $g$ be a Kähler metric of $X$ and $m$ be an arbitrary positive integer. For tangent vectors $\left\{e_{i}\right\}_{i=1}^{m}$ in $T_{X, p}$ at a point $p \in X$, the following equality holds:

$$
\sum_{i, j=1}^{m} R_{g}\left(e_{i}, \bar{e}_{i}, e_{j}, \bar{e}_{j}\right)=\frac{1}{2}\left\{\sum_{k=1}^{m} R_{g}\left(e_{k}, \overline{e_{k}}, e_{k}, \bar{e}_{k}\right)+\frac{1}{4^{m}} \sum_{\gamma \in I^{m}} R_{g}\left(\eta_{\gamma}, \bar{\eta}_{\gamma}, \eta_{\gamma}, \bar{\eta}_{\gamma}\right)\right\},
$$

where $I:=\{1,-1, \sqrt{-1},-\sqrt{-1}\}$ and $\eta_{\gamma}:=\sum_{k=1}^{m} \varepsilon_{k} e_{k}$ for $\gamma=\left(\varepsilon_{1}, \varepsilon_{2}, \ldots, \varepsilon_{m}\right) \in I^{m}$. In particular, if the holomorphic sectional curvature is semi-positive, then the left hand side is non-negative.

Lemma 2.2 ([Yan17, Lemma 4.1], [Yan18a, Lemma 6.1]). Let $g$ be a Kähler metric of $X$ and $V$ be a subspace of $T_{X, p}$ at a point $p \in X$. If a unit vector $x \in V$ minimizes the holomorphic sectional curvature $H_{g}$ on $V$, that it, it satisfies

$$
\min \left\{H_{g}([v]) \mid 0 \neq v \in V\right\}=H_{g}([x])
$$

then we have

$$
2 R_{g}(x, \bar{x}, w, \bar{w}) \geq\left(1+\left|\langle x, w\rangle_{g}\right|^{2}\right) R_{g}(x, \bar{x}, x, \bar{x})
$$

for any unit vector $w \in V$. In particular, if the holomorphic sectional curvature is semipositive, a minimizer $x$ of $H_{g}$ on $V$ satisfies that

$$
R_{g}(x, \bar{x}, w, \bar{w}) \geq 0
$$

for any tangent vector $w \in V$.

The case where $V$ in Lemma 2.2 coincides with the whole tangent space $T_{X, p}$ is proved in [Yan18a, Lemma 6.1]. It is easy to see that the same argument as in [Yan18a, Lemma 6.1] works even in the case of $V$ being a subspace of $T_{X, p}$, and thus we omit the proof of Lemma 2.2. Note that we essentially use the assumption that $g$ is a Kähler metric in the proof of the above lemmas.

2.2. $\mathrm{RC}$ positivity and vanishing theorems. In this subsection, we recall the notion of $\mathrm{RC}$ positivity of vector bundles introduced in [Yan18a]. Moreover we generalize a vanishing theorem for RC-negative vector bundles to treat RC semi-positivity in the proof of Theorem 1.4 .

Definition 2.3 (RC positivity, [Yan18a]). A hermitian vector bundle $(E, g)$ on a complex manifold $X$ is called $R C$ positive (resp. $R C$ negative) at $p \in X$, if for any non-zero vector $b \in E_{p}$ there exists a tangent vector $v \in T_{X, p}$ such that

$$
R_{g}(v, \bar{v}, b, \bar{b})>0 \quad(\text { resp. }<0) \text { at } p .
$$


Further $(E, g)$ is simply called $R C$ positive (resp. $R C$ negative), if it is RC positive (resp. $\mathrm{RC}$ negative) at every point in $X$.

Remark 2.4. A hermitian line bundle is RC positive if and only if it is $(n-1)$-positive (that is, it admits a hermitian metric whose Chern curvature has at least one positive eigenvalue everywhere). Recall that $n$ is the dimension of $X$.

If a line bundle admits a hermitian metric satisfying the condition of RC positivity (that is, $(n-1)$-positivity), then a partial vanishing theorem of Andreotti-Grauert type holds. The converse implication (which was first asked in [DPS96]) was established in [Yan19] (see also [Mat13], [Ott12], [Tot13] for related topics). In summary, we have the following result:

Theorem 2.5 ([Yan19], cf. [DPS96], [Mat13]). Let L be a line bundle on a compact complex manifold $X$ of dimension $n$. Then the following conditions are equivalent:

- The dual line bundle $L^{\vee}$ is not pseudo-effective.

- L admits a hermitian metric with $R C$ positive curvature $((n-1)$-positive curvature).

Moreover, when $X$ is a smooth projective variety, the above conditions are equivalent to the following condition:

- $L$ is $(n-1)$-ample, that is, for any coherent sheaf $\mathcal{F}$ on $X$, there is a positive integer $m_{0}$ such that

$$
H^{n}\left(X, \mathcal{F} \otimes L^{\otimes m}\right)=0 \text { for any } m \geq m_{0} .
$$

In the proof of Theorem 1.4, we need the following vanishing theorem for partially RC-negative vector bundles, which can be seen as a generalization of [Yan18a, Theorem $3.5]$.

Theorem 2.6. Let $E$ and $F$ be vector bundles on a compact complex manifold $X$, and let $t: F \rightarrow E$ be an injective sheaf morphism. (Note that we use the same notation for vector bundles and locally free sheaves). Assume that there is a (proper) subvariety $V$ on $X$ with the following properties:

- E admits a hermitian metric $g$ (defined on $X$ ) such that for any point $p \in X \backslash V$ and for any non-zero vector

$$
b \in \operatorname{Im}\left(t: F_{p} \rightarrow E_{p}\right) \subset E_{p},
$$

there is a tangent vector $v \in T_{X, p}$ satisfying

$$
R_{g}(v, \bar{v}, b, \bar{b})<0 \text {. }
$$

Then we have

$$
H^{0}\left(X, F \otimes \mathcal{I}_{V}\right)=0
$$

where $\mathcal{I}_{V}$ is the ideal sheaf associated to the subvariety $V$.

Proof. For a given section $s$ in $H^{0}\left(X, F \otimes \mathcal{I}_{V}\right)$, we consider the section $\widetilde{s}$ of $E$ obtained from the induced injective morphism

$$
H^{0}\left(X, F \otimes \mathcal{I}_{V}\right) \subset H^{0}(X, F) \hookrightarrow H^{0}(X, E) .
$$


It is sufficient to check that $\widetilde{s}$ is identically zero on $X$. We take a point $p_{0} \in X$ that attains the maximum value of the (point-wise) norm $|\widetilde{s}|_{g}$. We may assume that $\widetilde{s}\left(p_{0}\right)$ is a non-zero vector in $E$. The section $\widetilde{s}$ is identically zero on $V$ by the construction of $\widetilde{s}$, and thus $p_{0}$ is outside the subvariety $V$.

Now we have the following equality:

$$
\sqrt{-1} \partial \bar{\partial}|\widetilde{s}|_{g}^{2}=\sqrt{-1}\left\langle D^{\prime} \widetilde{s}, D^{\prime} \widetilde{s}\right\rangle_{g}-\left\langle\sqrt{-1} \Theta_{g}(\widetilde{s}), \widetilde{s}\right\rangle_{g},
$$

where $D^{\prime}$ is the $(1,0)$-part of the Chern connection. The left hand side is a semi-negative $(1,1)$-form at $p_{0}$ by the choice of $p_{0}$. On the other hand, since

$$
b:=\widetilde{s}\left(p_{0}\right) \in \operatorname{Im}\left(t: F_{p_{0}} \rightarrow E_{p_{0}}\right) \subset E_{p_{0}}
$$

is a non-zero vector, we can find a tangent vector $v \in T_{X, p_{0}}$ such that

$$
\left\langle\sqrt{-1} \Theta_{g}(v, \bar{v})(\widetilde{s}), \widetilde{s}\right\rangle_{g}=R_{g}(v, \bar{v}, b, \bar{b})<0 \text { at } p_{0}
$$

by the assumption. This is a contradiction.

\section{Proof of Theorem 1.4 And its Corollaries}

This section is devoted to the proof of Theorem 1.4 and its corollary.

Proof of Theorem 1.4. The proof can be divided into two steps. The main idea comes from Step 1, in which we consider the situation of $\phi$ being a smooth morphism. In Step 2 , by modifying this idea in Step 1 to treat an arbitrary meromorphic map $\phi$, we prove a generalization of Theorem 1.4 for a projective variety $Y$ with canonical singularities.

Step 1 (The case of $\phi$ being a smooth morphism). In this step, we show only that $K_{Y}$ is not an ample line bundle under the situation that $\phi: X \rightarrow Y$ in Theorem 1.4 is a smooth morphism.

Let $g$ be a Kähler metric of $X$ with semi-positive holomorphic sectional curvature. The surjective bundle morphism

$$
\left(T_{X}, g\right) \stackrel{d \phi_{*}}{\longrightarrow}\left(\phi^{*} T_{Y}, h\right)
$$

can be obtained from the differential map $d \phi_{*}$. We remark that the above morphism is surjective as a bundle morphism since $\phi$ is a smooth morphism. Further the Kähler metric $g$ and the above bundle morphism induce the hermitian metric $h$ on the pull-back $\phi^{*} T_{Y}$ of the tangent bundle $T_{Y}$ of $Y$. We put $m:=\operatorname{dim} Y, \Omega_{X}:=T_{X}^{\vee}$, and $\Omega_{Y}:=T_{Y}^{\vee}$. We obtain the injective bundle morphism

$$
\left(\phi^{*} \Lambda^{m} \Omega_{Y}=\phi^{*} K_{Y}, \Lambda^{m} h^{\vee}\right) \stackrel{\Lambda^{m} d \phi^{*}}{\longrightarrow}\left(\Lambda^{m} \Omega_{X}, \Lambda^{m} g^{\vee}\right)
$$

by taking the dual vector bundle and the $m$-th exterior product, where $\bullet \vee$ denotes the dual bundle of vector bundles or the dual hermitian metric. Then the following claim follows from Royden's lemma. 
Claim 3.1. For any point $p \in X$ and any non-zero vector

$$
b \in \operatorname{Im}\left(\Lambda^{m} d \phi^{*}: \phi^{*} K_{Y, p} \stackrel{\Lambda^{m} d \phi^{*}}{\longrightarrow} \Lambda^{m} \Omega_{X, p}\right) \subset \Lambda^{m} \Omega_{X, p} \text { at } p,
$$

there exists a tangent vector $v \in T_{X, p}$ with the following property:

$$
\text { - } d \phi_{*}(v) \neq 0 \text { in } T_{Y, \phi(p)} \text {. } \quad \text { } R_{\Lambda^{m} g^{\vee}}(v, \bar{v}, b, \bar{b}) \leq 0 \text {. }
$$

Remark 3.2. Even if $\phi$ is not a smooth morphism on the whole space $X$, the argument below still works for a point $p \in X$ at which $\phi$ is smooth. This argument will be used again in Step 2.

Proof of Claim 3.1. For a given point $p \in X$, we choose an orthonormal basis $\left\{e_{i}\right\}_{i=1}^{n}$ of the tangent space $T_{X, p}$ at $p$ such that $\left\{d \phi_{*}\left(e_{i}\right)\right\}_{i=1}^{m}$ is also an orthonormal basis of $\phi^{*} T_{Y, p}=T_{Y, \phi(p)}$. Here $n$ denotes the dimension of $X$. We define $V$ by the subspace

$$
V:=\operatorname{Spn}\left\langle\left\{\left\{e_{i}\right\}_{i=1}^{m}\right\}\right\rangle \subset T_{X, p}
$$

spanned by $\left\{e_{i}\right\}_{i=1}^{m}$ and the vector $a$ by

$$
a:=e_{1} \wedge e_{2} \wedge \cdots \wedge e_{m} \in \Lambda^{m} V \subset \Lambda^{m} T_{X, p}
$$

It is sufficient for the proof to find a tangent vector $v \in T_{X, p}$ such that

$$
d \phi_{*}(v) \neq 0 \text { in } T_{Y, \phi(p)} \quad \text { and } \quad R_{\Lambda^{m} g}(v, \bar{v}, a, \bar{a}) \geq 0
$$

for a non-zero vector $a=e_{1} \wedge e_{2} \wedge \cdots \wedge e_{m} \in \Lambda^{m} V$, since the image $\operatorname{Im}\left(\Lambda^{m} d \phi^{*}\right) \subset \Lambda^{m} \Omega_{X, p}$ is spanned by the vector $e_{1}^{\vee} \wedge e_{2}^{\vee} \wedge \cdots \wedge e_{m}^{\vee}$, where $\left\{e_{i}^{\vee}\right\}_{i=1}^{n}$ denotes the dual basis of $\left\{e_{i}\right\}_{i=1}^{n}$.

For an arbitrary index $i \in\{1,2, \ldots, m\}$, we put

$$
A_{i}:=R_{\Lambda^{m} g}\left(e_{i}, \bar{e}_{i}, a, \bar{a}\right)
$$

and for simplicity we put

$$
B_{i}(\bullet):=\sqrt{-1} \Theta_{g}\left(e_{i}, \bar{e}_{i}\right)(\bullet) \in \operatorname{End}\left(T_{X, p}\right) .
$$

Then we can easily check that

$$
\sqrt{-1} \Theta_{\Lambda^{m} g}\left(e_{i}, \bar{e}_{i}\right)(a)=\sum_{j=1}^{m} e_{1} \wedge \cdots \wedge B_{i}\left(e_{j}\right) \wedge \cdots \wedge e_{m}
$$


PROJECTIVE MANIFOLDS WITH SEMI-POSITIVE HOLOMORPHIC SECTIONAL CURVATURE 9 by the definition of $\sqrt{-1} \Theta_{\Lambda^{m} g}$ (see equality (2.1)). A straightforward computation yields

$$
\begin{aligned}
A_{i} & =\left\langle\sqrt{-1} \Theta_{\Lambda^{m} g}\left(e_{i}, \bar{e}_{i}\right)(a), a\right\rangle_{\Lambda^{m} g} \\
& =\sum_{j=1}^{m}\left\langle e_{1} \wedge \cdots \wedge B_{i}\left(e_{j}\right) \wedge \cdots \wedge e_{m}, e_{1} \wedge e_{2} \wedge \cdots \wedge e_{m}\right\rangle_{\Lambda^{m} g} \\
& =\sum_{j=1}^{m}\left\langle B_{i}\left(e_{j}\right), e_{j}\right\rangle_{g} \\
& =\sum_{j=1}^{m} R_{g}\left(e_{i}, \bar{e}_{i}, e_{j}, \bar{e}_{j}\right) .
\end{aligned}
$$

By Royden's lemma (see Lemma 2.1) and the assumption of the holomorphic sectional curvature being semi-positive, we can obtain

$$
\sum_{i=1}^{m} A_{i}=\sum_{i, j=1}^{m} R_{g}\left(e_{i}, \bar{e}_{i}, e_{j}, \bar{e}_{j}\right)=\frac{1}{2}\left\{\sum_{k=1}^{m} R_{g}\left(e_{k}, \overline{e_{k}}, e_{k}, \overline{e_{k}}\right)+\frac{1}{4^{m}} \sum_{\gamma \in I^{m}} R_{g}\left(\eta_{\gamma}, \bar{\eta}_{\gamma}, \eta_{\gamma}, \overline{\eta_{\gamma}}\right)\right\} \geq 0 .
$$

Therefore it can be seen that

$$
A_{i_{0}}=R_{\Lambda^{m} g}\left(e_{i_{0}}, \overline{i_{0}}, a, \bar{a}\right) \geq 0
$$

for some $i_{0} \in\{1,2, \ldots, m\}$. By the choice of the orthonormal basis, the vector $d \phi_{*}\left(e_{i_{0}}\right)$ is a non-zero vector in $T_{Y, \phi(p)}$. This completes the proof.

In the rest of this step, we show that $K_{Y}$ is not an ample line bundle by using Claim 3.1 and Theorem 2.5. Since $\left(\phi^{*} K_{Y}, \Lambda^{m} h^{\vee}\right)$ is a subbundle of $\left(\Lambda^{m} \Omega_{X}, \Lambda^{m} g^{\vee}\right)$ as hermitian vector bundles, we have

$$
\sqrt{-1} \Theta_{\Lambda^{m} h^{\vee}}(v, \bar{v})|c|_{\Lambda^{m} h^{\vee}}^{2}=R_{\Lambda^{m} h^{\vee}}(v, \bar{v}, c, \bar{c}) \leq R_{\Lambda^{m} g^{\vee}}(v, \bar{v}, b, \bar{b})
$$

for any vector $v \in T_{X}$ and a non-zero vector $c \in \phi^{*} K_{Y}$, where $b:=\Lambda^{m} d \phi^{*}(c) \in \Lambda^{m} \Omega_{X}$. For an arbitrary point $p \in X$, we can find a tangent vector $v \in T_{X, p}$ such that

$$
d \phi_{*}(v) \neq 0 \text { in } T_{Y, \phi(p)} \quad \text { and } \quad \sqrt{-1} \Theta_{\Lambda^{m} h^{\vee}}(v, \bar{v}) \leq 0
$$

by Claim 3.1 and $|c|_{\Lambda^{m} h^{\vee}} \neq 0$.

On the other hand, if $K_{Y}$ is assumed to be an ample line bundle, there is a smooth hermitian metric $H$ on $K_{Y}$ with the (strictly) positive curvature $\sqrt{-1} \Theta_{H}>0$. Then it can be shown that the line bundle $\phi^{*} K_{Y}$ is RC negative. Indeed, for the hermitian metric on $\phi^{*} K_{Y}$ defined by

$$
\left(\Lambda^{m} h^{\vee}\right)^{2} \cdot\left(\phi^{*} H\right)^{-1}
$$


the curvature $\sqrt{-1} \Theta_{\left(\Lambda^{m} h^{\vee}\right)^{2} \cdot\left(\phi^{*} H\right)^{-1}}$ satisfies that

$$
\begin{aligned}
\sqrt{-1} \Theta_{\left(\Lambda^{m} h^{\vee}\right)^{2} \cdot\left(\phi^{*} H\right)^{-1}}(v, \bar{v}) & =2 \sqrt{-1} \Theta_{\left(\Lambda^{m} h^{\vee}\right)}(v, \bar{v})-\phi^{*} \sqrt{-1} \Theta_{H}(v, \bar{v}) \\
& \leq-\sqrt{-1} \Theta_{H}\left(d \phi_{*}(v), d \phi_{*}^{-}(v)\right) \\
& <0
\end{aligned}
$$

for the tangent vector $v \in T_{X, p}$ obtained in Claim 3.1. The last inequality follows from $d \phi_{*}(v) \neq 0$ and $\sqrt{-1} \Theta_{H}>0$.

The dual bundle $\phi^{*} K_{Y}^{\vee}$ is RC positive, and thus $\phi^{*} K_{Y}$ is not pseudo-effective by Theorem 2.5. It contradicts to the assumption that $K_{Y}$ is an ample line bundle.

Step 2 (The proof of Theorem 1.4). In this step, we prove the following statement by modifying the idea in Step 1, which is a generalization of Theorem 1.4.

Theorem 3.3. Let $X$ be a compact Kähler manifold with semi-positive holomorphic sectional curvature, and let $\phi: X \rightarrow Y$ be a dominant meromorphic map from $X$ to a projective variety $Y$ with at most canonical singularities Then $K_{Y}$ is not a big line bundle.

Proof of Theorem 3.3. Even if $\phi$ is a morphism, the morphism (3.1) is not injective as a bundle morphism (since the rank of the linear map defined on fibers may not be constant), but it induces the injective sheaf morphism between locally free sheaves $\phi^{*} K_{Y}$ and $\Lambda^{m} \Omega_{X}$. From now on, we interchangeably use the words "vector bundles" and "locally free sheaves", and we use the same notation for the induced sheaf morphism. The main differences from Step 1 are that we have to treat the indeterminacy locus and that we can not obtain the induced metric on $\phi^{*} K_{Y}$ since the morphism (3.1) is not a bundle morphism. To overcome these difficulties, we apply Theorem 2.6 instead of Theorem 2.5.

We first take a resolution $\tau: \bar{X} \rightarrow X$ of the indeterminacy locus $B$ of $\phi$ such that it passes through a resolution $\mu: \bar{Y} \rightarrow Y$ of singularities of $Y$. The morphisms $\varphi$ and $\bar{\phi}$ are defined by the following diagram:

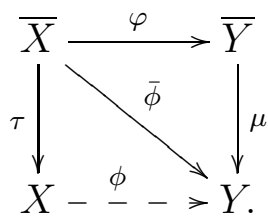

For a contradiction, we assume that $K_{Y}$ is a big line bundle. It can be seen that there exist a very ample line bundle $A$ on $Y$ and an effective Cartier divisor $E$ on $Y$ such that $K_{Y}^{\otimes m_{0}}=A \otimes E$ holds for some $m_{0}>0$ by Kodaira lemma. We define the "pull-backs" of the Cartier divisors $K_{Y}^{\otimes m_{0}}$ and $A$ by

$$
\phi^{*} K_{Y}^{\otimes m_{0}}:=\tau_{*} \bar{\phi}^{*}\left(K_{Y}^{\otimes m_{0}}\right) \quad \text { and } \quad \phi^{*} A=\tau_{*} \bar{\phi}^{*} A .
$$

Let $\left\{t_{i}\right\}_{i \in I}$ be a basis of $H^{0}(Y, A)$. The sections $\left\{t_{i}\right\}_{i \in I}$ determine the smooth hermitian metric $H$ on $A$. Indeed, the hermitian metric $H$ on $A$ can be defined to be

$$
|e|_{H}^{2}:=\frac{|e|^{2}}{\sum_{i \in I}\left|t_{i}\right|^{2}}
$$


PROJECTIVE MANIFOLDS WITH SEMI-POSITIVE HOLOMORPHIC SECTIONAL CURVATURE 11

for every vector $e \in A$. It follows that the Chern curvature $\sqrt{-1} \Theta_{H}$ is a positive $(1,1)$-form on the non-singular locus $Y_{\text {reg }}:=Y \backslash Y_{\text {sing }}$ of $Y$ since $A$ is a very ample line bundle on $Y$.

Similarly, the pull-backs $\left\{\phi^{*} t_{i}\right\}_{i \in I}$ of the sections $\left\{t_{i}\right\}_{i \in I}$ under $\phi$, which are sections of $\phi^{*} A$, also determine the "singular" hermitian metric on $\phi^{*} A$, which we denote by the notation $\phi^{*} H$ (see [Dem] for singular hermitian metrics). The section $\phi^{*} t_{i}$ obtained from the pull-back of $t_{i}$ is identically zero on the indeterminacy locus $B$ (otherwise it contradicts to the fact that $B$ is the indeterminacy locus and $A$ is very ample). Hence we can see that $\phi^{*} H$ has analytic singularities along the indeterminacy locus $B$.

We consider a point $p \in X$ such that $\phi(p) \in Y_{\text {reg }}$ and $\phi$ is a morphism at $p$. It can be seen that $\phi^{*} H$ is smooth at $p$ and that $\sqrt{-1} \Theta_{\phi^{*} H}=\phi^{*} \sqrt{-1} \Theta_{H}$ holds at $p$. Therefore, by Claim 3.1 (see also Remark 3.2) and Step 1, we can obtain the following claim:

Claim 3.4. We consider a point $p \in X$ such that $\phi(p) \in Y_{\text {reg }}$ and $\phi$ is a morphism at $p$. Let $\ell$ be a positive integer. Then, for any non-zero vector

$$
b \in \operatorname{Im}\left(S^{\ell}\left(\Lambda^{m} d \phi^{*}\right): \phi^{*} K_{Y, p}^{\otimes \ell} \stackrel{S^{\ell}\left(\Lambda^{m} d \phi^{*}\right)}{\longrightarrow} S^{\ell}\left(\Lambda^{m} \Omega_{X, p}\right)\right) \subset S^{\ell}\left(\Lambda^{m} \Omega_{X, p}\right) \text { at } p,
$$

there exists a tangent vector $v \in T_{X, p}$ with the following property:

$$
\text { - } d \phi_{*}(v) \neq 0 \text { in } T_{Y, \phi(p)} \text {. } \quad \text { - } R_{S^{\ell}\left(\Lambda^{m} g^{\vee}\right)}(v, \bar{v}, b, \bar{b}) \leq 0 .
$$

Moreover, for such a point $p$ and a non-zero vector

$$
b \in \operatorname{Im}\left(S^{\ell}\left(\Lambda^{m} d \phi^{*}\right) \otimes i d: \phi^{*} K_{Y, p}^{\otimes \ell} \otimes \phi^{*} A_{p}^{\vee} \stackrel{S^{\ell}\left(\Lambda^{m} d \phi^{*}\right) \otimes i d}{\longrightarrow} S^{\ell}\left(\Lambda^{m} \Omega_{X, p}\right) \otimes \phi^{*} A_{p}^{\vee}\right) \text { at } p,
$$

there exists a tangent vector $v \in T_{X, p}$ such that

$$
R_{S^{\ell}\left(\Lambda^{m} g^{\vee}\right) \otimes \phi^{*} H^{\vee}}(v, \bar{v}, b, \bar{b})<0 .
$$

Proof of Claim 3.4. We choose an orthonormal basis $\left\{e_{i}\right\}_{i=1}^{n}$ of $T_{X, p}$ at $p$ such that $\left\{d \phi_{*}\left(e_{i}\right)\right\}_{i=1}^{m}$ is also an orthonormal basis of $\phi^{*} T_{Y, p}$. Note that the morphism $\phi$ is a smooth morphism at $p$ (otherwise there is no non-zero vector in the image). Let $V \subset T_{X, p}$ be the subspace $V:=\operatorname{Spn}\left\langle\left\{\left\{e_{i}\right\}_{i=1}^{m}\right\}\right\rangle$ spanned by $\left\{e_{i}\right\}_{i=1}^{m}$ and $a^{\odot \ell}$ be the vector defined by

$$
a^{\odot \ell}:=\left(e_{1} \wedge e_{2} \wedge \cdots \wedge e_{m}\right)^{\odot \ell} \in S^{\ell}\left(\Lambda^{m} V\right) \subset S^{\ell}\left(\Lambda^{m} T_{X, p}\right) .
$$

For any $i \in\{1,2, \ldots, m\}$, we obtain

$$
\begin{aligned}
\sqrt{-1} \Theta_{S^{\ell}\left(\Lambda^{m} g\right)}\left(e_{i}, \bar{e}_{i}\right)\left(a^{\odot \ell}\right) & =\sum_{k=1}^{\ell} a \odot \cdots \odot \sqrt{-1} \Theta_{\Lambda^{m} g}\left(e_{i}, \bar{e}_{i}\right)(a) \odot \cdots \odot a \\
& =\ell \sqrt{-1} \Theta_{\Lambda^{m} g}\left(e_{i}, \bar{e}_{i}\right)(a) \odot a^{\odot \ell-1}
\end{aligned}
$$

from equality (2.2). Hence it can be shown that

$$
\begin{aligned}
R_{S^{\ell}\left(\Lambda^{m} g\right)}\left(e_{i}, \bar{e}_{i}, a^{\odot \ell}, a^{\bar{\odot} \ell}\right) & =\left\langle\sqrt{-1} \Theta_{S^{\ell}\left(\Lambda^{m} g\right)}\left(e_{i}, \bar{e}_{i}\right)\left(a^{\odot \ell}\right), a^{\odot \ell}\right\rangle_{S^{\ell}\left(\Lambda^{m} g\right)} \\
& =\ell\left\langle\sqrt{-1} \Theta_{\Lambda^{m} g}\left(e_{i}, \bar{e}_{i}\right)(a) \odot a^{\odot \ell-1}, a^{\odot \ell}\right\rangle_{S^{\ell}\left(\Lambda^{m} g\right)} \\
& =\ell\left\langle\sqrt{-1} \Theta_{\Lambda^{m} g}\left(e_{i}, \bar{e}_{i}\right)(a), a\right\rangle_{\Lambda^{m} g}
\end{aligned}
$$


By Royden's lemma (see Lemma 2.1) and the proof of Claim 3.1, we can easily check that the right hand side is non-negative for some $i_{0} \in\{1,2, \ldots, m\}$. This leads to the first conclusion.

We will check the latter conclusion. The vector $b$ in the claim can be written as $b=$ $b_{1}^{\odot \ell} \otimes b_{2}$, where $b_{1}$ is a vector in the image of

$$
\phi^{*} K_{Y, p} \stackrel{\Lambda^{m} d \phi^{*}}{\longrightarrow} \Lambda^{m} \Omega_{X, p}
$$

and $b_{2}$ is a vector in $\phi^{*} A_{p}^{\vee}$. Then, for any tangent vector $v \in T_{X, p}$, we obtain

$$
\begin{aligned}
& R_{S^{\ell}\left(\Lambda^{m} g^{\vee}\right) \otimes \phi^{*} H^{\vee}}(v, \bar{v}, b, \bar{b}) \\
= & R_{S^{\ell}\left(\Lambda^{m} g^{\vee}\right)}\left(v, \bar{v}, b_{1}^{\odot \ell}, b_{1}^{\odot \ell}\right)\left|b_{2}\right|_{\phi^{*} H^{\vee}}^{2}+\left|b_{1}^{\odot \ell}\right|_{S^{\ell}\left(\Lambda^{m} g^{\vee}\right)}^{2} R_{\phi^{*} H^{\vee}}\left(v, \bar{v}, b_{2}, \overline{b_{2}}\right) \\
= & R_{S^{\ell}\left(\Lambda^{m} g^{\vee}\right)}\left(v, \bar{v}, b_{1}^{\odot \ell}, b_{1}^{\overline{\odot \ell}}\right)\left|b_{2}\right|_{\phi^{*} H^{\vee}}^{2}+\left|b_{1}^{\odot \ell}\right|_{S^{\ell}\left(\Lambda^{m} g^{\vee}\right)}^{2}\left|b_{2}\right|_{\phi^{*} H^{\vee}}^{2} \sqrt{-1} \Theta_{\phi^{*} H^{\vee}}(v, \bar{v})
\end{aligned}
$$

from (2.3) and (3.3). When the tangent vector $v$ satisfies the first conclusion, we can see that

$$
R_{S^{\ell}\left(\Lambda^{m} g^{\vee}\right)}\left(v, \bar{v}, b_{1}^{\odot \ell}, b_{1}^{\overline{\odot \ell}}\right) \leq 0 \quad \text { and } \quad \sqrt{-1} \Theta_{\phi^{*} H^{\vee}}(v, \bar{v})=-\sqrt{-1} \Theta_{H}\left(d \phi_{*}(v), d \phi_{*}^{-}(v)\right)<0
$$

from $d \phi_{*}(v) \neq 0$ and $\sqrt{-1} \Theta_{H}>0$. This completes the proof.

In the rest of this step, we will finish the proof of Theorem 3.3 by applying the above claim and Theorem 2.6. For a sufficiently divisible integer $\ell=k m_{0}$, we consider the formula

$$
\mu^{*} K_{Y}^{\otimes \ell}=K_{\bar{Y}}^{\otimes \ell} \otimes F^{\otimes-\ell}
$$

Here $F$ is the effective divisor since $Y$ has at most canonical singularities. Then we obtain the injective sheaf morphisms

$$
\begin{gathered}
\bar{\phi}^{*} A^{\otimes k-1} \stackrel{\otimes t_{0}}{\longrightarrow} \bar{\phi}^{*} A^{\otimes k-1} \otimes \bar{\phi}^{*} E^{\otimes k}=\bar{\phi}^{*} K_{Y}^{\otimes \ell} \otimes \bar{\phi}^{*} A^{\vee}=\varphi^{*}\left(K_{\bar{Y}}^{\otimes \ell} \otimes F^{\otimes-\ell}\right) \otimes \bar{\phi}^{*} A^{\vee} \\
\stackrel{\otimes t}{\longrightarrow} \varphi^{*} K_{\bar{Y}}^{\otimes \ell} \otimes \bar{\phi}^{*} A^{\vee} \stackrel{S^{\ell}\left(\Lambda^{m} d \varphi^{*}\right) \otimes i d}{\longrightarrow} S^{\ell}\left(\Lambda^{m} \Omega \bar{X}\right) \otimes \bar{\phi}^{*} A^{\vee}
\end{gathered}
$$

where $\otimes t$ (resp. $\left.t_{0}\right)$ is the multiplication map defined by the natural section $t$ (resp. $t_{0}$ ) of the effective divisor $\varphi^{*} F^{\otimes \ell}=\ell \varphi^{*} F\left(\operatorname{resp} . \bar{\phi}^{*} E^{\otimes k}=k \bar{\phi}^{*} E\right)$. Further we have

$$
\tau_{*}\left(S^{\ell}\left(\Lambda^{m} \Omega_{\bar{X}}\right) \otimes \bar{\phi}^{*} A^{\vee}\right)=S^{\ell}\left(\Lambda^{m} \Omega_{X}\right) \otimes \phi^{*} A^{\vee} \text { and } \tau_{*}\left(\bar{\phi}^{*} A^{\otimes k-1}\right)=\phi^{*} A^{\otimes k-1}
$$

by the definition. Therefore we obtain the injective sheaf morphism

$$
\phi^{*} A^{\otimes k-1} \longrightarrow S^{\ell}\left(\Lambda^{m} \Omega_{X}\right) \otimes \phi^{*} A^{\vee} .
$$


By taking the pull-back under $\bar{\phi}$, chasing the injective morphisms induced by (3.4), and using equality (3.5), we obtain the following diagram:

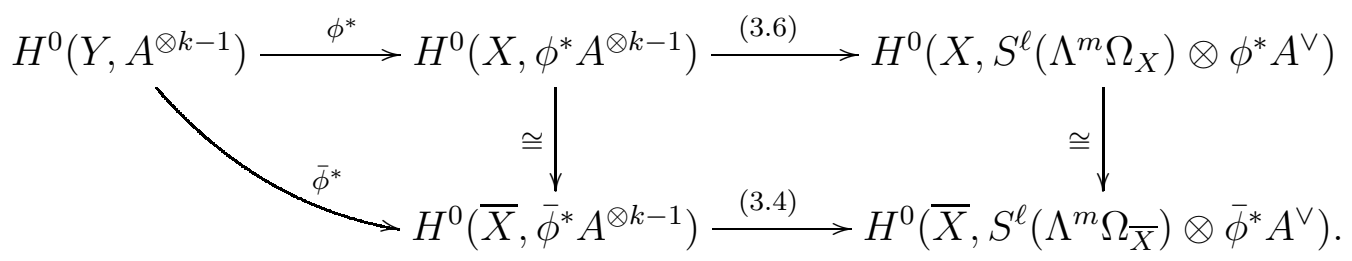

By taking a sufficiently large integer $k$, we can choose a non-zero section $s$ in $H^{0}\left(Y, A^{\otimes k-1}\right)$ such that $s$ is identically zero on the singular locus $Y_{\text {sing }}$, by ampleness of $A$. We consider the non-zero section

$$
\widetilde{s} \in H^{0}\left(X, S^{\ell}\left(\Lambda^{m} \Omega_{X}\right) \otimes \phi^{*} A^{\vee}\right)
$$

obtained from the above injective morphisms. The metric $\phi^{*} H$ is a singular hermitian metric, but it has analytic singularities, and thus $\phi^{*} H^{\vee}$ can be seen locally as a smooth function (which is identically zero on $B$ ). Therefore the point-wise norm $|\widetilde{s}|_{S^{\ell}\left(\Lambda^{m} g^{\vee}\right) \otimes \phi^{*} H^{\vee}}$ of $\widetilde{s}$ is a smooth function on $X$. Thus we can take a maximizer $p_{0} \in X$ of this norm, that is, $p_{0} \in X$ satisfies that

$$
\max _{X}|\widetilde{s}|_{S^{\ell}\left(\Lambda^{m} g^{\vee}\right) \otimes \phi^{*} H^{\vee}}=|\widetilde{s}|_{S^{\ell}\left(\Lambda^{m} g^{\vee}\right) \otimes \phi^{*} H^{\vee}}\left(p_{0}\right) .
$$

It can be seen that that $\widetilde{s}$ is identically zero on $B$ since $\widetilde{s}$ is obtained via the pull-back under $\phi$. In particular, the point $p_{0}$ is outside $B$. Further it follows that $\widetilde{s}$ is identically zero over $Y_{\text {sing }}$ by the choice of $s$. Therefore we can easily see that the same argument as in Theorem 2.6 works. (The only difference is that $\phi^{*} H$ is a singular hermitian metric, but it is smooth on a neighborhood of $p_{0}$.) Indeed, by applying equality $(2.4)$ to the non-zero vector $b:=\widetilde{s}\left(p_{0}\right)$, we can conclude that $\widetilde{s}$ is identically zero thanks to Claim 3.4 . This is a contradiction.

Compared to Step 1, the difficulty in Step 2 is to treat the singular locus $Y_{\text {sing }}$ and the indeterminacy locus $B$. The key point in the proof is that the indeterminacy locus $B$ is automatically killed and the singular locus $Y_{\text {sing }}$ is also killed by the zero locus of the section $\widetilde{s}$.

As we mentioned in Remark ??, by using the above method, Conjecture 1.2 can be solved if $Y$ admits a good minimal model $Y \rightarrow Y_{\min }$. The main idea is to kill the singular locus $Z_{\text {sing }}$ and the non-smooth locus of a morphism $f: Y_{\min } \rightarrow Z$ by the zero locus of the section $\widetilde{s}$, where $Z$ is the canonical model of $Y_{\text {min }}$. For this purpose, the following lemma, which can be seen as a generalization of Claim 3.1 and Claim 3.4, plays an important role, but we omit the detail.

Lemma 3.5. Let $X, Y$, and $Z$ be complex manifolds. For morphisms $\phi: X \rightarrow Y$ and $f: Y \rightarrow Z$, we assume that $\psi:=f \circ \phi: X \rightarrow Z$ is a smooth morphism at $p \in X$. Further let $g$ be a Kähler metric of $X$ with the semi-positive holomorphic sectional curvature $H_{g}$. 
We put $m:=\operatorname{dim} Z$. We consider the induced metric $\Lambda^{m} g^{\vee}$ on $\Lambda^{m} \Omega_{X}$. Then, for any non-zero vector vector

$$
b \in \operatorname{Im}\left(\Lambda^{m} d \phi^{*}: \phi^{*} K_{Y, p} \stackrel{\Lambda^{m} d \phi^{*}}{\longrightarrow} \Lambda^{m} \Omega_{X, p}\right) \subset \Lambda^{m} \Omega_{X, p} \text { at } p,
$$

there exists a tangent vector $v \in T_{X, p}$ with the following properties:

$$
\text { - } d \psi_{*}(v) \neq 0 \text { in } T_{Z, \psi(p)} \text {. } \quad \bullet R_{\Lambda^{m} g^{\vee}}(v, \bar{v}, b, \bar{b}) \leq 0 .
$$

Moreover let $(A, H)$ be a smooth hermitian metric on $Z$ with the positive curvature $\sqrt{-1} \Theta_{H}>$ 0 . Then, for any non-zero vector

$$
b \in \operatorname{Im}\left(S^{\ell}\left(\Lambda^{m} d \phi^{*}\right) \otimes i d: \phi^{*}\left(K_{Y, p}^{\otimes \ell}\right) \otimes \psi^{*} A_{p}^{\vee} \stackrel{S^{\ell}\left(\Lambda^{m} d \phi^{*}\right) \otimes i d}{\longrightarrow} S^{\ell}\left(\Lambda^{m} \Omega_{X, p}\right) \otimes \psi^{*} A_{p}^{\vee}\right),
$$

there exists a tangent vector $v \in T_{X, p}$ such that

$$
R_{S^{\ell}\left(\Lambda^{m} g^{\vee}\right) \otimes \psi^{*} H^{\vee}}(v, \bar{v}, b, \bar{b})<0 .
$$

At the end of this paper, we prove Corollary 1.5.

Proof of Corollary 1.5. For a compact Kähler manifold with semi-positive holomorphic sectional curvature $H_{g}$, we can show that $X$ admits a finite étale cover by a complex torus or $K_{X}$ is not pseudo-effective. Indeed, when the holomorphic sectional curvature is identically zero, then $X$ admits a finite étale cover by a complex torus (see [HLW16, Proposition 2.2], [Ber66], [Igu54]). When it is not identically zero, we consider the scalar curvature $S$ of the Kähler metric $g$. Then we have

$$
\int_{X} c_{1}\left(K_{X}\right) \wedge \omega_{g}^{n-1}=-\frac{1}{\pi n} \int_{X} S \omega_{g}^{n},
$$

where $\omega_{g}$ is the Kähler form associated to $g$. The value of $S$ at a point $p \in X$ can be written as the integral of the holomorphic sectional curvature over the projective space $\mathbb{P}\left(T_{X, p}^{\vee}\right)$ (see [Ber66]). Therefore the right hand side is negative by the assumption that $H_{g}([v])>0$ holds for some tangent vector $v$. In particular, the canonical bundle $K_{X}$ is not pseudo-effective.

We consider a compact Kähler surface $X$ such that the holomorphic sectional curvature is not identically zero. Then, by the above argument, we can see that $K_{X}$ is not pseudoeffective. It is known that a compact complex surface such that $K_{X}$ is not pseudo-effective is a rational surface, or a minimal surface of class VII, or a ruled surface over a curve of genus $\geq 1$ by the classification of compact complex surfaces. However a minimal surface of class VII is not Kähler, and thus we can conclude that $X$ is rationally connected or a ruled surface over a curve of genus $\geq 1$. In the case where $X$ is a ruled surface, the genus of the base is less than or equal to one by Theorem 1.4. Therefore the base of a ruled surface with semi-positive holomorphic sectional curvature is an elliptic curve. 
PROJECTIVE MANIFOLDS WITH SEMI-POSITIVE HOLOMORPHIC SECTIONAL CURVATURE 15

\section{REFERENCES}

[BDPP13] S. Boucksom, J.-P. Demailly, M. Păun, T. Peternell, The pseudo-effective cone of a compact Kähler manifold and varieties of negative Kodaira dimension, J. Algebraic Geom. 22 (2013), no. 2, 201-248.

[Ber66] M. Berger, Sur les variétés d'Einstein compactes, Comptes Rendus de la IIIe Réunion du Groupement des Mathématiciens d'Expression Latine (Namur, 1965) pp. 35-55 Librairie Universitaire, Louvain (1966).

[Cam92] F. Campana, Connexité rationnelle des variétés de Fano, Ann. Sci. École Norm. Sup. (4) 25 (1992), no. 5, 539-545.

[CG71] J. Cheeger, D. Gromoll, The splitting theorem for manifolds of nonnegative Ricci curvature, J. Differential Geom. 6 (1971), 119-128.

[CG72] J. Cheeger, D. Gromoll, On the structure of complete manifolds of nonnegative curvature, Ann. of Math., 96 (1972), 413-443.

[DT19] S, Diverio, S. Trapani, Quasi-negative holomorphic sectional curvature and positivity of the canonical bundle, J. Differential Geom. 111 (2019), no. 2, 303-314.

[Dem] J.-P. Demailly, Analytic methods in algebraic geometry, Surveys of Modern Mathematics, 1, International Press, Somerville, Higher Education Press, Beijing, (2012).

[DPS96] J.-P. Demailly, T. Peternell, M. Schneider, Holomorphic line bundles with partially vanishing cohomology, Proceedings of the Hirzebruch 65 Conference on Algebraic Geometry (Ramat Gan, 1993), 165-198, Israel Math. Conf. Proc., 9, Bar-Ilan Univ 1996.

[GHS03] T. Graber, J. Harris, J. Starr, Families of rationally connected varieties, J. Amer. Math. Soc. 16 (2003), no. 1, 57-67.

[HW15] G. Heier, B. Wong, On projective Kähler manifolds of partially positive curvature and rational connectedness, Preprint, arXiv:1509.02149v1.

[HLW10] G. Heier, S. S. Y. Lu, B. Wong, On the canonical line bundle and negative holomorphic sectional curvature, Math. Res. Lett. 17 (2010), no. 6, 1101-1110.

[HLW16] G. Heier, S. S. Y. Lu, B. Wong, Kähler manifolds of semi-negative holomorphic sectional curvature, J. Differential Geom. 104 (2016), no. 3, 419-441.

[HLWZ17] G. Heier, S. S. Y. Lu, B. Wong, F. Zheng, Reduction of manifolds with semi-negative holomorphic sectional curvature, Preprint, arXiv:1705.00605v1.

[HSW81] A. Howard, B. Smyth, H. Wu, On compact Kähler manifolds of nonnegative bisectional curvature I and II, Acta Math. 147 (1981), no. 1-2, 51-70

[Igu54] J. Igusa, On the structure of a certain class of Kaehler varieties, Amer. J. Math. 76, (1954), 669-678.

[Nak] N. Nakayama, Zariski decomposition and abundance, MSJ Memoirs, 14. Mathematical Society of Japan, Tokyo, (2004).

[KoMM92] J. Kollár, Y. Miyaoka, S. Mori, Rationally connected varieties, J. Algebraic Geom. 1 (1992), no. 3, 429-448.

[Mat13] S. Matsumura, Asymptotic cohomology vanishing and a converse to the Andreotti-Grauert theorem on surfaces, Ann. Inst. Fourier (Grenoble) 63 (2013), no. 6, 2199-2221.

[Mat18a] S. Matsumura, On morphisms of compact Kähler manifolds with semi-positive holomorphic sectional curvature, Preprint, arXiv:1809.08859v1.

[Mat18b] S. Matsumura, On projective manifolds with semi-positive holomorphic sectional curvature, Preprint, arXiv:1811.04182v1.

[Mok88] N. Mok, The uniformization theorem for compact Kähler manifolds of nonnegative holomorphic bisectional curvature, J. Differential Geom. 27 (1988), no. 2, 179-214.

[Ott12] J. Ottem, Ample subvarieties and q-ample divisors, Adv. Math. 229 (2012), no. 5, 2868-2887. 
[Roy80] H. L. Royden, The Ahlfors-Schwarz lemma in several complex variables, Comment. Math. Helv. 55 (1980), no. 4, 547-558.

[Tot13] B. Totaro, Line bundles with partially vanishing cohomology, J. Eur. Math. Soc. (JEMS) 15 (2013), no. $3,731-754$.

[TY15] V. Tosatti, X. Yang, An extension of a theorem of Wu-Yau, J. Differential Geom. 107 (2017), no. 3, 573-579.

[Yan16] X. Yang, Hermitian manifolds with semi-positive holomorphic sectional curvature, Math. Res. Lett. 23 (2016), no. 3, 939-952.

[Yan17] X. Yang, Big vector bundles and complex manifolds with semi-positive tangent bundles, Math. Ann. 367 (2017), no. 1-2, 251-282.

[Yan18a] X. Yang, RC-positivity, rational connectedness and Yau's conjecture, Camb. J. Math. 6 (2018), no. 2, 183-212.

[Yan18b] X. Yang, RC-positive metrics on rationally connected manifolds, preprint, available at arXiv:1807.03510v2.

[Yan18c] X. Yang, RC-positivity, vanishing theorems and rigidity of holomorphic maps, preprint, available at arXiv:1807.02601v2.

[Yan19] X. Yang, A partial converse to the Andreotti-Grauert theorem, Compos. Math. 155 (2019), no. 1, 89-99.

[Yau82] S.-T. Yau, Problem section, Seminar on Differential Geometry, 669-706, Ann. of Math. Stud., 102, Princeton Univ. Press, Princeton, N.J, (1982).

[WY16] D. Wu, S.-T. Yau, Negative holomorphic curvature and positive canonical bundle, Invent. Math. 204 (2016), no. 2, 595-604.

Mathematical Institute, Tohoku University, 6-3, Aramaki Aza-Aoba, Aoba-ku, Sendai 980-8578, JAPAN.

Email address: mshinichi-math@tohoku.ac.jp, mshinichi0@gmail.com 